TRANSACTIONS OF THE

AMERICAN MATHEMATICAL SOCIETY

Volume 355, Number 12, Pages 4737-4753

S 0002-9947(03)03247-1

Article electronically published on July 24, 2003

\title{
A COMPACTIFICATION OF OPEN VARIETIES
}

\author{
YI HU
}

\begin{abstract}
In this paper we prove a general method to compactify certain open varieties by adding normal crossing divisors. This is done by showing that blowing up along an arrangement of subvarieties can be carried out. Important examples such as Ulyanov's configuration spaces and complements of arrangements of linear subspaces in projective spaces, etc., are covered. Intersection ring and (nonrecursive) Hodge polynomials are computed. Furthermore, some general structures arising from the blowup process are also described and studied.
\end{abstract}

\section{INTRODUCTION AND THE MAIN THEOREMS}

Throughout the paper, the base field is assumed to be algebraically closed.

Let $S$ be a partially ordered set (poset). The rank of $s \in S$ is the maximum of the lengths of all the chains that end up at $s$. A minimal element is of rank 0 . The rank of $S$ is the maximum of the lengths of all chains. Let $S_{\leq r}$ be the subposet of elements of rank $\leq r$. All posets in this paper are partially ordered by inclusion unless otherwise stated.

Two smooth closed subvarieties $U$ and $V$ of a smooth variety $W$ are said to intersect cleanly if the scheme-theoretic intersection $U \cap V$ is smooth and $T(U \cap V)=$ $T(U) \cap T(V)$ for their tangent spaces.

Theorem 1.1. Let $X^{0}$ be an open subset of a nonsingular algebraic variety $X$. Assume that $X \backslash X^{0}$ can be decomposed as a union $\bigcup_{i \in I} D_{i}$ of closed irreducible subvarieties such that

(1) $D_{i}$ is smooth;

(2) $D_{i}$ and $D_{j}$ meet cleanly;

(3) $D_{i} \cap D_{j}=\emptyset$ or a disjoint union of $D_{l}$.

The set $\mathcal{D}=\left\{D_{i}\right\}_{i}$ is then a poset. Let $k$ be the rank of $\mathcal{D}$. Then there is a sequence of well-defined blowups

$$
\mathrm{Bl}_{\mathcal{D}} X \rightarrow \mathrm{Bl}_{\mathcal{D}_{\leq k-1}} X \rightarrow \ldots \rightarrow \mathrm{Bl}_{\mathcal{D}_{\leq 0}} X \rightarrow X
$$

where $\mathrm{Bl}_{\mathcal{D}_{\leq 0}} X \rightarrow X$ is the blowup of $X$ along $D_{i}$ of rank 0 , and, inductively, $\mathrm{Bl}_{\mathcal{D}_{\leq r}} X \rightarrow \mathrm{Bl}_{\mathcal{D}_{\leq r-1}} X$ is the blowup of $\mathrm{Bl}_{\mathcal{D}_{r-1}} X$ along the proper transforms of $D_{j}$ of rank $r$, such that

(1) $\mathrm{Bl}_{\mathcal{D}} X$ is smooth;

(2) $\mathrm{Bl}_{\mathcal{D}} X \backslash X^{0}=\bigcup_{i \in I} \widetilde{D}_{i}$ is a divisor with normal crossings;

Received by the editors November 14, 2000.

2000 Mathematics Subject Classification. Primary 14C05; Secondary 05C30, 14N20. 
(3) $\widetilde{D}_{i_{1}} \cap \ldots \cap \widetilde{D}_{i_{n}}$ is nonempty if and only if $D_{i_{1}} \ldots D_{i_{n}}$ form a chain in the poset $\mathcal{D}$. Consequently, $\widetilde{D}_{i}$ and $\widetilde{D}_{j}$ meet if and only if $D_{i}$ and $D_{j}$ are comparable.

Definition 1.2. The set $\mathcal{D}$ is called an arrangement of smooth subvarieties. $\mathrm{Bl}_{\mathcal{D}} X$ is referred to as the blowup of $X$ along the arrangement $\mathcal{D}$ of subvarieties. When condition (3) of Theorem 1.1] is replaced by:

$\left(3^{\prime}\right) D_{i} \cap D_{j}=\emptyset$ or $D_{l}$ for some $l$, then $\mathcal{D}$ is called a simple arrangement of smooth subvarieties.

This work naturally extends the previous works of Fulton-MacPherson ([1]), MacPherson-Procesi ([6]) and Ulyanov ([7]). Our main theorem was especially inspired by Ulyanov's paper ([7]).

Any collection of (affine) linear subspaces $\left\{H_{i}\right\}$ in $\mathbb{P}^{n}$ (or $\mathbb{C}^{n}$ ) induces a simple arrangement of smooth subvarieties by taking all possible nonempty intersections (subspace arrangement). Theorem 1.1 applies to such a situation. A smooth curve of higher degree and a general line in $\mathbb{P}^{2} \subset \mathbb{P}^{n}(n>2)$ necessarily meet in several distinct points. Hence it is useful to include as well nonsimple arrangements of subvarieties.

More sophisticated and important examples are needed to situate Theorem 1.1 in particular cases, followed by stating certain general structures arising from the construction of the blowup $\mathrm{Bl}_{\mathcal{D}} X$.

- Configuration spaces. Consider $X^{n}$. Let $\Delta_{i j}$ be the subset of all points whose $i$-th and $j$-th coordinates coincide. Let $\Delta$ be the set of all possible intersections of $\Delta_{i j}$. $\Delta$ satisfies the arrangement conditions. We will call $\Delta$ the diagonal arrangement.

Corollary 1.3 (Ulyanov [7]). $\mathrm{Bl}_{\Delta}\left(X^{n}\right)$ is a symmetri] smooth projective compactification of $X^{n} \backslash \bigcup \Delta$ by adding smooth divisors with normal crossings.

Ulyanov also proved that $X\langle n\rangle:=\mathrm{Bl}_{\Delta}\left(X^{n}\right)$ dominates $X[n]$, the FultonMacPherson configuration space ([1]). $X[n]$ is not an instance of blowups along arrangement of subvarieties (cf. Definition 1.21).

- Space of holomorphic maps. Let $N_{d}\left(\mathbb{P}^{n}\right)$ be the space of $(n+1)$-tuples $\left(f_{0}, \ldots, f_{n}\right)$ modulo homothety where $f_{i}$ are homogeneous polynomials of degree $d$ in two variables and $N_{d}^{0}\left(\mathbb{P}^{n}\right)$ is the open subset such that $f_{0}, \ldots, f_{n}$ have no common zeros. $N_{d}^{0}\left(\mathbb{P}^{n}\right)$ is naturally identified with the space $M_{d}^{0}\left(\mathbb{P}^{n}\right)$ of holomorphic maps of degree $d$ from $\mathbb{P}^{1}$ to $\mathbb{P}^{n}$.

For any integer $0 \leq d_{0} \leq d$ and an arbitrary partition $\tau=\sum_{j} d_{j}$ of $d-d_{0}$, let

$$
N_{d_{0}, \tau}:=\left\{\left[\sigma_{0} \prod_{j>0}\left(a_{j} w_{0}-b_{j} w_{1}\right)^{d_{j}} \ldots \sigma_{n} \prod_{j>0}\left(a_{j} w_{0}-b_{j} w_{1}\right)^{d_{j}}\right]\right\} \subset N_{d}
$$

where $\sigma_{j}$ are homogeneous polynomials of degree $d_{0}$ and $\left\{\left[a_{j}, b_{j}\right]\right\}_{j}$ are unordered points in $\mathbb{P}^{1}$.

The collection $\mathcal{N}=\left\{N_{\pi, d_{0}}\right\}$ is however not an arrangement of smooth subvarieties in $N_{d}$, thanks to an important observation by Sean Keel who saved me from embarrassment. For, some strata $N_{\pi, d_{0}}$ may have singularities along lower strata. But the minimal ones are smooth so that the first step of the iterated blowups can be carried out. The hope is that after the first step, the singularieties of the strata

\footnotetext{
${ }^{1}$ That is, $\Sigma_{n}=\operatorname{Aut}\{1, \ldots, n\}$ acts on it.
} 
on the second level are resolved and get separated so that the next step of the iterated blowups can also be carried out. It calls for further investigation to see if the process can indeed be executed step by step to obtain a good compactification $\mathrm{Bl}_{\mathcal{N}} N_{d}\left(\mathbb{P}^{n}\right)$ of the space $N_{d}^{0}\left(\mathbb{P}^{n}\right)$ of holomorphic maps.

- GIT. Theorem 1.1 coupled with a compatible group action yields an instance of Theorem 1.1 in Geometric Invariant Theory. Roughly, it says that blowing up along an arrangement descends to blowing up of any GIT quotient along an induced arrangement. As a particular case, we recover Kirwan's partial desingularization of singular GIT quotients. See Corollary 7.3 in 7.

We now return to the general situation.

- Proper transforms and exceptional divisors. Of useful computational value is that in each stage of the blowups, $\mathrm{Bl}_{\mathcal{D}_{\leq r}} X \rightarrow X$, the proper transforms of $D_{i}$ and exceptional divisors are special instances of Theorem 1.1 and all are concisely described using posets induced from $\mathcal{D}$.

Theorem 1.4 (Proper transforms). Let $\mathcal{D}^{r+1}$ be the set of proper transforms in $\mathrm{Bl}_{\mathcal{D}_{\leq r}} X$ of $D_{i}$ of rank $\geq r+1$. Then

(1)

$$
\mathcal{D}^{r+1}=\left\{\mathrm{Bl}_{\left(\mathcal{D}_{<D_{i}}\right)_{\leq r}} D_{i}: \operatorname{rank}\left(D_{i}\right) \geq r+1\right\}
$$

where $\mathcal{D}_{<D_{i}}$ denotes the subposet of the elements less than $D_{i}$, and

$$
\mathcal{D}_{\leq 0}^{r+1}=\left\{\mathrm{Bl}_{\left(\mathcal{D}_{<D_{i}}\right)_{\leq r}} D_{i}: \operatorname{rank}\left(D_{i}\right)=r+1\right\}
$$

(2) $\mathcal{D}^{r+1}$ is an arrangement of smooth subvarieties in $\mathrm{Bl}_{\mathcal{D}_{\leq r}} X$.

Corollary 1.5. With the above notations, $\mathrm{Bl}_{\mathcal{D}} X$ can be expressed as iterated blowups along (explicit) disjoint centers.

$$
\mathrm{Bl}_{\mathcal{D}} X=\mathrm{Bl}_{\mathcal{D}_{\leq 0}^{k}} \mathrm{Bl}_{\mathcal{D}_{\leq 0}^{k-1}} \ldots \mathrm{Bl}_{\mathcal{D}_{\leq 0}^{1}} \mathrm{Bl}_{\mathcal{D}_{\leq 0}} X
$$

Corollary 1.6. The intermediate blowup

$$
\mathrm{Bl}_{\mathcal{D}_{\leq r}} X \rightarrow X
$$

is an instance of the theorem when the arrangement of subvarieties is the subarrangement $\mathcal{D}_{\leq r}$. In particular,

$$
\mathrm{Bl}_{\mathcal{D}_{\leq r}} X=\mathrm{Bl}_{\mathcal{D}_{\leq 0}^{r}} \mathrm{Bl}_{\mathcal{D}_{\leq 0}^{r-1}} \ldots \mathrm{Bl}_{\mathcal{D}_{\leq 0}^{1}} \mathrm{Bl}_{\mathcal{D}_{\leq 0}} X
$$

Theorem 1.7 (Exceptional divisors). Let $\mathcal{E}^{r+1}$ be the set of all the exceptional divisors of $\mathrm{Bl}_{\mathcal{D}_{\leq r}} X \rightarrow X$. Then $\mathcal{E}^{r+1}$ consists of

(1) for each $D_{i}$ of rank $r$,

$$
E_{i}^{r+1}=\mathbb{P}\left(N_{D_{i}^{r} / \mathrm{Bl}_{\mathcal{D}_{\leq r-1} X}}\right),
$$

which are also exceptional divisors of the blowup $\mathrm{Bl}_{\mathcal{D}_{\leq r}} X \rightarrow \mathrm{Bl}_{\mathcal{D}_{\leq r-1}} X$, where $\mathrm{Bl}_{\mathcal{D}_{\leq-1}} X:=X$;

(2) for each $D_{i}$ of rank $m<r$,

$$
E_{i}^{r+1}=\mathrm{Bl}_{\left\{D_{j}^{m+1} \cap E_{i}^{m+1}: D_{j}>D_{i}\right\}_{\leq r-m}} E_{i}^{m+1} .
$$

Note that by (1), $E_{i}^{m+1}=\mathbb{P}\left(N_{D_{i}^{m} / \mathrm{Bl}_{\mathcal{D}_{\leq m-1}} X}\right)$. 
The expression of $E_{i}^{r+1}$ relies on the proper transform $D_{i}^{m}$ and is the blowup

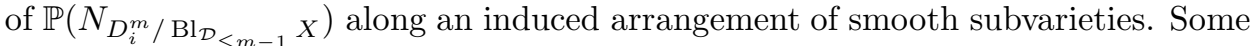
topological calculations on $N_{D_{i}^{m} / \mathrm{Bl}_{\mathcal{D}_{\leq m-1}} X}$ can be reduced to $N_{D_{l} / X}$ for $D_{l} \leq D_{i}$ (e.g., 速).

- Intersection rings. An embedding $U \hookrightarrow W$ is called a Lefschetz embedding if the restriction map $A^{\bullet}(W) \rightarrow A^{\bullet}(U)$ is surjective. Let $J_{U / W}$ be the kernel of $A^{\bullet}(W) \rightarrow A^{\bullet}(U)$ and let $P_{U / W}$ be a Chern polynomial for the normal bundle $N_{U / W}(\$ 4)$.

A simple arrangement $\mathcal{D}$ is called regular if for any $D_{l}<D_{i}$ there is $D_{j}>D_{l}$ such that $D_{l}=D_{i} \cap D_{j}$. That is, any $D_{l}$ is an intersection of maximal $D_{i}$. All the previously mentioned examples are regular.

Theorem 1.8. Let $\mathcal{D}$ be a regular simple arrangement of subvarieties. Assume that all inclusions $D_{i} \subset D_{j}$ and $D_{i} \subset X$ are Lefschetz embeddings. Then the Chow ring $A^{\bullet}\left(\mathrm{Bl}_{\mathcal{D}} X\right)$ is isomorphic to the polynomial ring

$$
A^{\bullet}(X)\left[T_{1}, \ldots, T_{N}\right] / I
$$

where $T_{i}$ corresponds $t D^{2} D_{i}$ and $I$ is the ideal generated by

(1) $T_{i} \cdot T_{j}$ for incomparable $D_{i}$ and $D_{j}$;

(2) $J_{D_{i} / X} \cdot T_{i}$ for all $i$;

(3) $P_{D_{i} / X}\left(-\sum_{D_{j} \leq D_{i}} T_{j}\right)$ for all $i$.

This theorem can be directly applied to the intermediate stage $\mathrm{Bl}_{\mathcal{D}_{<r}} X$ by Corollary 1.6, to the proper transforms $D_{i}^{r}$ by Theorem 1.4, and to the exceptional divisors by Theorem 1.7

- Hodge and Poincaré polynomials. The concise presentations of $\mathrm{Bl}_{\mathcal{D}} X$, the intermediate stage $\mathrm{Bl}_{\mathcal{D}_{\leq r}} X$ and the proper transform $D_{i}^{r}$ of $D_{i}$ in every stage allow one to derive a concise nonrecursive formula for the Hodge (Poincaré) polynomial of $\mathrm{Bl}_{\mathcal{D}} X$.

Let $\mathbf{e}(W)(\mathbf{P}(W))$ be the Hodge (Poincaré) polynomial in two (resp. one) variables $u$ and $v$ (resp. $t$ ) of a smooth projective variety $W$.

\section{Theorem 1.9.}

$$
\begin{gathered}
\mathbf{e}\left(\mathrm{Bl}_{\mathcal{D}} X\right)=\mathbf{e}(X)+\sum_{\substack{D_{i_{1}}<\ldots<D_{i_{r+1}} \\
D_{i_{r+1}}:=X}} \mathbf{e}\left(D_{i_{1}}\right) \prod_{j=1}^{r} \frac{(u v)^{\operatorname{dim} D_{i_{j+1}}-\operatorname{dim} D_{i_{j}}-u v}}{u v-1} . \\
\mathbf{P}\left(\mathrm{Bl}_{\mathcal{D}} X\right)=\mathbf{P}(X)+\sum_{\substack{D_{i_{1}}<\ldots<D_{i_{r+1}} \\
D_{i_{r+1}}:=X}} \mathbf{P}\left(D_{i_{1}}\right) \prod_{j=1}^{r} \frac{t^{2 \operatorname{dim} D_{i_{j+1}}-2 \operatorname{dim} D_{i_{j}}-t^{2}}}{t^{2}-1} .
\end{gathered}
$$

This formula immediately applies to the intermediate stage $\mathrm{Bl}_{\mathcal{D}_{\leq r}} X$, the proper transform $D_{i}^{r}$ and the exceptional divisor $E_{i}^{r}$.

Consider $X\langle n\rangle$. The index set of the diagonal arrangement $\Delta$ is the set of all partitions $\pi$ of $[n]=\{1, \ldots, n\}$ except the largest trivial partition $\mathbf{1}_{[n]}=1 \cup \ldots \cup n$. The subvariety $\Delta_{\pi} \subset \Delta$ is the set of all points, any two of whose coordinates

\footnotetext{
${ }^{2}$ More precisely and geometrically, $T_{i}$ corresponds to the exceptional divisor $E_{i}^{r+1}$, where $r=\operatorname{rank}\left(D_{i}\right)$. See the proof in 4 See also Theorem 1.7 (1) for the description of $E_{i}^{r+1}$.
} 
coincide whenever their indexes belong to the same block of $\pi$. Let $\rho(\pi)$ be the number of blocks of $\pi$. Then $\Delta_{\pi} \cong X^{\rho(\pi)}$.

Corollary 1.10.

$$
\begin{gathered}
\mathbf{e}(X\langle n\rangle)=\mathbf{e}(X)^{n}+\sum_{\substack{\pi_{i_{1}}<\ldots<\pi_{i_{r+1}} \\
\pi_{i_{r+1}}:=\mathbf{1}_{[n]}}} \mathbf{e}(X)^{\rho\left(\pi_{i_{1}}\right)} \prod_{j=1}^{r} \frac{(u v)^{\operatorname{dim} X\left(\rho\left(\pi_{i_{j+1}}\right)-\rho\left(\pi_{i_{j}}\right)\right)}-u v}{u v-1} . \\
\mathbf{P}(X\langle n\rangle)=\mathbf{P}(X)^{n}+\sum_{\substack{\pi_{i_{1}}<\ldots<\pi_{i_{r+1}} \\
\pi_{i_{r+1}}:=\mathbf{1}_{[n]}}} \mathbf{P}(X)^{\rho\left(\pi_{i_{1}}\right)} \prod_{j=1}^{r} \frac{\left(t^{2}\right)^{\operatorname{dim} X\left(\rho\left(\pi_{i_{j+1}}\right)-\rho\left(\pi_{i_{j}}\right)\right)}-t^{2}}{t^{2}-1} .
\end{gathered}
$$

Corollary 1.11. Let $\mathcal{H}=\left\{H_{i}\right\}$ be an arrangement of linear subspaces of $\mathbb{P}^{n}$. Then

$$
\begin{aligned}
& \mathbf{e}\left(\mathrm{Bl}_{\mathcal{H}} \mathbb{P}^{n}\right)=\frac{1}{u v} \sum_{\substack{H_{i_{0}}<H_{i_{1}}<\ldots<H_{i_{r+1}} \\
\emptyset:=H_{i_{0}}, H_{i_{r+1}}:=X}} \prod_{j=0}^{r} \frac{(u v)^{\operatorname{dim} H_{i_{j+1}}-\operatorname{dim} H_{i_{j}}-u v}}{u v-1}, \\
& \mathbf{P}\left(\mathrm{Bl}_{\mathcal{H}} \mathbb{P}^{n}\right)=\frac{1}{t^{2}} \sum_{\substack{H_{i_{0}}<H_{i_{1}}<\ldots<H_{i_{r+1}} \\
\emptyset:=H_{i_{0}}, H_{i_{r+1}}:=X}} \prod_{j=0}^{r} \frac{t^{2 \operatorname{dim} H_{i_{j+1}}-2 \operatorname{dim} H_{i_{j}}}-t^{2}}{t^{2}-1},
\end{aligned}
$$

where $\operatorname{dim} \emptyset=-2$.

Take $n+2$ points of $\mathbb{P}^{n}$ in general linear position. They span $\left(\begin{array}{c}n+2 \\ 2\end{array}\right)$ hyperplanes. Let $\mathcal{H}_{n}$ be the induced simple arrangement. Then $\mathrm{Bl}_{\mathcal{H}_{n}} \mathbb{P}^{n}$ is isomorphic to $\bar{M}_{0, n+3}$. This example is due to Kapranov. The index set of $\mathcal{H}_{n}$ is the set of all subsets $S$ of $[n+2]$ such that $1 \leq|S| \leq n$.

\section{Corollary 1.12.}

$$
\begin{aligned}
& \mathbf{e}\left(\bar{M}_{0, n+3}\right)=\frac{1}{u v} \sum_{\substack{S_{i_{0}}<S_{i_{1}}<\ldots<S_{i_{r}}<S_{i_{r}+1} \\
\emptyset:=S_{i_{0}},\left|S_{i_{r+1}}\right|:=n+1}} \prod_{j=0}^{r} \frac{(u v)^{\left|S_{i_{j+1}}\right|-\left|S_{i_{j}}\right|}-u v}{u v-1}, \\
& \mathbf{P}\left(\bar{M}_{0, n+3}\right)=\frac{1}{t^{2}} \sum_{\substack{S_{i_{0}}<S_{i_{1}}<\ldots<S_{i_{r}}<S_{i_{r+1}} \\
\emptyset:=S_{i_{0}},\left|S_{i_{r+1}}\right|:=n+1}} \prod_{j=0}^{r} \frac{t^{2\left|S_{i_{j+1}}\right|-2\left|S_{i_{j}}\right|-t^{2}}}{t^{2}-1},
\end{aligned}
$$

where $|\emptyset|=-1$.

Keel computed these numbers, and furthermore he also computed the intersection ring (4]).

Corollary 1.13. Let $\mathcal{H}=\left\{H_{i}\right\}$ be an arrangement of linear subspaces of $\mathbb{C}^{n}$. Then

$$
\begin{aligned}
& \mathbf{e}\left(\mathrm{Bl}_{\mathcal{H}} \mathbb{C}^{n}\right)=1+\frac{1}{u v} \sum_{\substack{H_{i_{1}}<\ldots<H_{i_{r}}<H_{i_{r+1}} \\
H_{i_{r+1}}:=\mathbb{C}^{n}}} \prod_{j=1}^{r} \frac{(u v)^{\operatorname{dim} H_{i_{j+1}}-\operatorname{dim} H_{i_{j}}-u v}}{u v-1} . \\
& \mathbf{P}\left(\mathrm{Bl}_{\mathcal{H}} \mathbb{C}^{n}\right)=1+\frac{1}{t^{2}} \sum_{\substack{H_{i_{1}}<\ldots<H_{i_{r}}<H_{i_{r+1}} \\
H_{i_{r+1}}:=\mathbb{C}^{n}}} \prod_{j=1}^{r} \frac{t^{2 \operatorname{dim} H_{i_{j+1}}-2 \operatorname{dim} H_{i_{j}}}-t^{2}}{t^{2}-1} .
\end{aligned}
$$


Finally, it needs to be pointed out that the general procedure of blowing up along arrangements can be extended to some singular cases as well. This may be necessary in certain applications (see $\S \S 6$ and 7 ).

The paper is structured as follows. $\S 2$ provides proofs of the statements in this introduction on the structures of the blowup along arrangement $\mathrm{Bl}_{\mathcal{D}} X$, the exceptional divisors $E_{i}^{r}$ and proper transforms $D_{i}^{r}$ of $D_{i}$. Some corollaries to the proofs are also drawn. $\S 3$ gives an alternative construction of $\mathrm{Bl}_{\mathcal{D}} X$ as the closure of the open subset. $\S 4$ proves the statement on the intersection ring of $\mathrm{Bl}_{\mathcal{D}} X$. $\S 5$ proves the formulas for Hodge and Poincaré polynomials as stated in this introduction. $\S 6$ is devoted to the spaces of holomorphic maps. $\S 7$ treats blowups of GIT quotients along induced arrangements.

I learned from Professor Fulton that Dylan Thurston (while still an undergraduate at Harvard) noticed several years ago that $X[n]$ could be constructed by a sequence of symmetric blowups - but one has to blow up along ideal sheaves. The point is that one can blow up along two smooth subvarieties that meet excessively in a smooth subvariety without first blowing up the small variety.

I wonder if $X[n]$ is the minimal symmetric compactification of the configuration space $X^{n} \backslash \bigcup \Delta$ by adding normal crossing divisors. I thank Fulton and MacPherson for their powerful original inspiring work [1]. This paper is dedicated to them.

\section{Proof of Theorems 1.1, 1.4, and 1.7}

Lemma 2.1. Let $U$ and $V$ be two smooth closed subvarieties of a smooth variety $W$ that intersect cleanly. Then

(1) the proper transforms of $U$ and $V$ in $\mathrm{Bl}_{U \cap V} W$ are disjoint;

(2) the proper transform of $V$ in $\mathrm{Bl}_{U} W$ is isomorphic to $\mathrm{Bl}_{U \cap V} V$;

(3) if $Z$ is a smooth subvariety of $U \cap V$, then the proper transforms of $U$ and $V$ in $\mathrm{Bl}_{Z} W$ intersect cleanly.

Proof. All follow from standard arguments.

Lemma 2.2 (Flag Blowup Lemma; [1] and [7). Let $V_{0}^{1} \subset V_{0}^{2} \subset \ldots \subset V_{0}^{s} \subset W$ be a flag of smooth subvarieties in a smooth algebraic variety $W_{0}$. For $k=1, \ldots, s$, define inductively: $W_{k}$ is the blowup of $W_{k-1}$ along $V_{k-1}^{k} ; V_{k}^{k}$ is the exceptional divisor in $W_{k}$; and $V_{k}^{i}, k \neq i$, is the proper transform of $V_{k-1}^{i}$ in $W_{k}$. Then the preimage of $V_{0}^{s}$ in the resulting variety $W_{s}$ is a normal crossing divisor $V_{s}^{1} \cup \ldots \cup V_{s}^{s}$.

Proof. See [7].

Proof of Theorem 1.1. Without the awkward but routine verification of the inductive proof, the construction goes quite transparently by the clarification as follows.

First, $\mathrm{Bl}_{\mathcal{D}_{\leq 0}} X \rightarrow X$ is the blow up of $X$ along the disjoint smooth subvarieties of $D_{i}$ of rank 0 .

Let $D_{j}^{1}$ be the proper transform of $D_{j}$ of rank $\geq 1$. By Lemma 2.1 (1), the proper transforms $D_{j}^{1}$ of $D_{j}$ of rank 1 are disjoint in $\mathrm{Bl}_{\mathcal{D}_{\leq 0}} X$. By Lemma 2.1 (2) and (3), all $D_{j}^{1}$ are smooth and intersect cleanly (or trivially). If $D_{i} \cap D_{j}=\coprod_{l} D_{l}$, then $D_{i}^{1} \cap D_{j}^{1}=\coprod_{\operatorname{rank}\left(D_{l}\right)>0} D_{l}^{1}$. Otherwise, $D_{i}^{1} \cap D_{j}^{1}=\emptyset$. This shows that

$$
\mathcal{D}^{1}=\left\{D_{j}^{1}=\operatorname{Bl}_{\mathcal{D}_{<D_{j}}} D_{j}: \operatorname{rank}\left(D_{j}\right) \geq 1\right\}
$$


is an arrangement of subvarieties in $\mathrm{Bl}_{\mathcal{D}_{\leq 0}} X$. Moreover,

$$
\mathcal{D}_{\leq 0}^{1}=\left\{D_{j}^{1}=\operatorname{Bl}_{\mathcal{D}_{<D_{j}}} D_{j}: \operatorname{rank}\left(D_{j}\right)=1\right\} .
$$

This makes the next step possible, which is essentially a repetition of the first step:

$$
\mathrm{Bl}_{\mathcal{D}_{\leq 1}} X=\mathrm{Bl}_{\mathcal{D}_{\leq 0}^{1}}\left(\mathrm{Bl}_{\mathcal{D}_{\leq 0}} X\right) \rightarrow \mathrm{Bl}_{\mathcal{D}_{\leq 0}} X
$$

Note that $\operatorname{rank}\left(\mathcal{D}^{1}\right)=\operatorname{rank}(\mathcal{D})-1$.

Let $\mathcal{D}^{2}$ be the proper transform of $D_{j}$ in $\mathrm{Bl}_{\mathcal{D}_{\leq 1}} X$ of rank $\geq 2$. The same reasoning as above shows that

$$
\mathcal{D}^{2}=\left\{D_{j}^{1}=\mathrm{Bl}_{\left(\mathcal{D}_{<D_{j}}\right) \leq 1} D_{j}: \operatorname{rank}\left(D_{j}\right) \geq 2\right\}
$$

is an arrangement of smooth subvarieties and

$$
\mathcal{D}_{\leq 0}^{2}=\left\{D_{j}^{1}=\mathrm{Bl}_{\left(\mathcal{D}_{<D_{j}}\right) \leq 1} D_{j}: \operatorname{rank}\left(D_{j}\right)=2\right\} .
$$

Blowing up subvarieties in $\mathcal{D}_{\leq 0}^{2}$, we obtain

$$
\mathrm{Bl}_{\mathcal{D}_{\leq 2}} X=\mathrm{Bl}_{\mathcal{D}_{\leq 0}^{2}}\left(\mathrm{Bl}_{\mathcal{D}_{\leq 1}} X\right) \rightarrow \mathrm{Bl}_{\mathcal{D}_{\leq 1}} X .
$$

Note that $\operatorname{rank}\left(\mathcal{D}^{2}\right)=\operatorname{rank}\left(\mathcal{D}^{1}\right)-1=\operatorname{rank}(\mathcal{D})-2$.

The above can be repeated until the subvarieties in the rank 0 poset $\mathcal{D}^{k}$ are blown up. That is, the resulting variety from the last step is the iterated blowup along smooth disjoint centers

$$
\mathrm{Bl}_{\mathcal{D}} X=\mathrm{Bl}_{\mathcal{D}_{\leq 0}^{k}} \mathrm{Bl}_{\mathcal{D}_{\leq 0}^{k-1}} \ldots \mathrm{Bl}_{\mathcal{D}_{\leq 0}^{1}} \mathrm{Bl}_{\mathcal{D}_{\leq 0}} X
$$

Statement (1) follows from this description.

If $D_{i} \cap D_{j} \neq \emptyset, D_{i}, D_{j}$, that is, $D_{i}$ and $D_{j}$ are incomparable, by Lemma 2.1 (1), their proper transforms become disjoint at the stage

$$
\mathrm{Bl}_{\mathcal{D}_{\leq r}} X \rightarrow X
$$

for $r=\max \left\{\operatorname{rank}\left(D_{l}\right): D_{l} \subset D_{i} \cap D_{j}\right\}$. Hence $\widetilde{D}_{i_{1}} \cap \ldots \cap \widetilde{D}_{i_{n}}$ is nonempty if and only if $D_{i_{1}}, \ldots, D_{i_{n}}$ form a chain in the poset $\mathcal{D}$. This proves statement (3).

Statement (2) then follows directly from the Flag Blowup Lemma. Here one needs to observe that for any maximal chain $D_{i_{1}}<\ldots<D_{i_{n}}$, by the above proof of (3), blowing up the proper transform of any $D_{j}$ which is not in the chain is irrelevant to the intersection $\widetilde{D}_{i_{1}} \cap \ldots \cap \widetilde{D}_{i_{n}}$. Hence the Flag Blowup Lemma applies.

We now draw an easy consequence. Let $\gamma$ be a chain

$$
D_{i_{1}}<\ldots<D_{i_{n}}
$$

and let $S_{\gamma}$ be the intersection $\widetilde{D}_{i_{1}} \cap \ldots \cap \widetilde{D}_{i_{n}}$. Set

$$
S_{\gamma}^{0}:=S_{\gamma} \backslash \bigcup_{\gamma^{\prime} \supset \gamma} S_{\gamma^{\prime}}
$$

We allow $\gamma=\emptyset$ and define $S_{\emptyset}:=X$; hence $S_{\emptyset}^{0}=X^{0}$. Then the normal crossing property implies that

Corollary 2.3. $\bigcup_{\gamma} S_{\gamma}^{0}$ is a Whitney stratification of $\mathrm{Bl}_{\mathcal{D}} X$ by locally closed smooth subvarieties. 
Proof of Theorem 1.4. (1) and (2) will be proved simultaneously by using induction on $r$.

When $r=0$ (the case for $\mathcal{D}^{1}$ ), the proof is contained in the proof of Theorem 1.1 .

Assume that statements (1) and (2) are valid for $\mathcal{D}^{r}$.

Consider the blowup $\mathrm{Bl}_{\mathcal{D}_{\leq 0}^{r}}\left(\mathrm{Bl}_{\mathcal{D}_{\leq r-1}} X\right) \rightarrow \mathrm{Bl}_{\mathcal{D}_{\leq r-1}} X$. $\mathcal{D}^{r+1}$ is the set of proper transforms $D_{i}^{r+1}$ of $D_{i}^{r}=\mathrm{Bl}_{\left(\mathcal{D}_{<D_{i}}\right) \leq r-1} D_{i} \in \mathcal{D}^{r}$ for $D_{i}$ of rank $\geq r+1$. Hence

$$
D_{i}^{r+1}=\mathrm{Bl}_{\left\{D_{j}^{r}: D_{j}<D_{i}, \operatorname{rank}\left(D_{j}\right)=r\right\}} \mathrm{Bl}_{\left(\mathcal{D}_{<D_{i}}\right) \leq r-1} D_{i}=\mathrm{Bl}_{\left(\mathcal{D}_{<D_{i}}\right)_{\leq r}} D_{i} .
$$

The reasoning in the proof of Theorem 1.3 for $\mathcal{D}^{1}$ to be an arrangement of smooth subvarieties can be applied to the blowup

$$
\mathrm{Bl}_{\mathcal{D}_{\leq 0}^{r}}\left(\mathrm{Bl}_{\mathcal{D}_{\leq r-1}} X\right) \rightarrow \mathrm{Bl}_{\mathcal{D}_{\leq r-1}} X
$$

to yield the same statement for $\mathcal{D}^{r+1}$.

This completes the proof.

Specializing to the diagonal arrangement of $X^{n}$, we draw a sample consequence. Let $\pi$ be a nontrivial partition of $[n]$. Then we have

Corollary 2.4. The proper transform $\Delta_{\pi}^{\rho(\pi)-1} \cong X\langle\rho(\pi)\rangle$.

Proofs of Corollaries 1.5 and 1.6. Corollary 1.5 is contained in the proof of Theorem 1.1 (This corollary does not logically depend on Theorem 1.4 but depends on the notations introduced there. To keep the introduction coherent, we put the statement after Theorem 1.4.)

Corollary 1.6 follows from essentially the same reason.

Proof of Theorem 1.7. (1) and (2) will be proved simultaneously by using induction on $r$.

When $r=0$ (the case for $\mathcal{E}^{1}$ ), consider the blowup $\mathrm{Bl}_{\mathcal{D}_{\leq 0}} X \rightarrow X$; the statements are standard.

Assume that the statement is valid for $\mathcal{E}^{r}$.

Consider the blowup $\mathrm{Bl}_{\mathcal{D}_{\leq 0}^{r}}\left(\mathrm{Bl}_{\mathcal{D}_{\leq r-1}} X\right) \rightarrow \mathrm{Bl}_{\mathcal{D}_{\leq r-1}} X$. The center of the blowup is $D_{i}^{r}$ for $D_{i}$ of rank $r$. Hence statement (1) is standard.

The rest of the exceptional divisors of $\mathrm{Bl}_{\mathcal{D}_{\leq r}} X \rightarrow X$ come from the proper transforms of $E_{i}^{r}$ for $D_{i}$ of rank $m \leq r-1$. Hence they are

$$
\begin{gathered}
E_{i}^{r+1}=\mathrm{Bl}_{\left\{E_{i}^{r} \cap D_{l}^{r}: D_{l}>D_{i} \operatorname{rank}\left(D_{l}\right)=r\right\}} E_{i}^{r} \\
=\mathrm{Bl}_{\left\{E_{i}^{r} \cap D_{l}^{r}: D_{l}>D_{i} \operatorname{rank}\left(D_{l}\right)=r\right\}}\left(\mathrm{Bl}_{\left\{D_{j}^{m+1} \cap E_{i}^{m+1}: D_{j}>D_{i}\right\}_{\leq r-1-m}} E_{i}^{m+1}\right) .
\end{gathered}
$$

Now observe that

$$
\left\{D_{j}^{m+1} \cap E_{i}^{m+1}: D_{j}>D_{i}\right\}_{\leq 0}^{r-m}=\left\{E_{i}^{r} \cap D_{l}^{r}: D_{l}>D_{i}, \operatorname{rank}\left(D_{l}\right)=r\right\} .
$$

Hence, by Theorem 1.1 (or its proof),

$$
E_{i}^{r+1}=\mathrm{Bl}_{\left\{D_{j}^{m+1} \cap E_{i}^{m+1}: D_{j}>D_{i}\right\}_{\leq r-m}} E_{i}^{m+1} .
$$




\section{3. $\mathrm{Bl}_{\mathcal{D}} X$ AS A CLOSURE}

Theorem 3.1. $\mathrm{Bl}_{\mathcal{D}} X$ is the closure of $X^{0}=X \backslash \bigcup_{i} D_{i}$ in

$$
X \times \prod_{i} \mathrm{Bl}_{D_{i}} X
$$

Proof. We will prove the following statement by induction.

$X_{r+1}=\mathrm{Bl}_{\mathcal{D}_{\leq r}} X$ is the closure of $X^{0}$ in $X \times \prod_{D_{i} \in \mathcal{D}_{\leq r}} \mathrm{Bl}_{D_{i}} X$.

When $r=0$, the statement is clear because $X_{1}$ is the blowup of $X$ along disjoint $D_{i} \in \mathcal{D}_{\leq 0}$ and is thus the same as the closure of the graph of the rational map $X \rightarrow \prod_{D_{i} \in \mathcal{D}_{\leq 0}}^{-} \mathrm{Bl}_{D_{i}} X$. Assume that the statement for $X_{r}$ is proved. $X_{r+1}$ is the blowup of $X_{r}$ along the minimal subvarieties in $\mathcal{D}_{\leq 0}^{r}$. This is interpreted as blowing up the ideal of the sheaf

$$
\prod_{\operatorname{rank} D_{i}=r} \mathcal{I}\left(D_{i}^{r}\right)
$$

where $\mathcal{I}(D)$ is the ideal sheaf for a closed subvariety $D$. Denote by $p_{r}$ the projection $X_{r} \rightarrow X$ and by $\left(p_{r}^{*} \mathcal{I}\left(D_{j}\right)\right)$ the ideal generated by the pull-back $p_{r}^{*} \mathcal{I}\left(D_{j}\right)$. Then by Corollary 1.6 and Theorem 1.1 .

$$
\left(p_{r}^{*} \mathcal{I}\left(D_{i}\right)\right)=\mathcal{I}\left(D_{i}^{r}\right) \prod_{D_{j}<D_{i}} \mathcal{I}\left(E_{j}^{r}\right) .
$$

Observe that $\prod_{D_{j}<D_{i}} \mathcal{I}\left(E_{j}^{r}\right)$ is an invertible sheaf. Hence

$$
\prod_{\operatorname{rank} D_{i}=r} \mathcal{I}\left(D_{i}^{r}\right) \text { and } \prod_{\operatorname{rank} D_{i}=r}\left(p_{r}^{*} \mathcal{I}\left(D_{j}\right)\right.
$$

differ by a multiple of an invertible sheaf. Therefore blowing up

$$
\prod_{\operatorname{rank} D_{i}=r} \mathcal{I}\left(D_{i}^{r}\right)
$$

is the same as taking the closure of the graph of the rational map $X_{r} \rightarrow$ $\prod_{\text {rank } D_{i}=r} \mathrm{Bl}_{D_{i}} X$. By the inductive assumption, it is the same as the closure of $X^{0}$ in

$$
X \times \prod_{D_{i} \in \mathcal{D}_{\leq r-1}} \mathrm{Bl}_{D_{i}}(X) \times \prod_{\operatorname{rank}\left(D_{i}\right)=r} \mathrm{Bl}_{D_{i}} X .
$$

This finishes the inductive proof.

The statement of the theorem is the case when $r=\operatorname{rank}(\mathcal{D})$.

\section{INTERSECTION RING OF $\mathrm{Bl}_{\mathcal{D}}$}

For any inclusion $U \hookrightarrow W$ of a smooth closed subvariety $U$ in a smooth variety $W, J_{U / W}$ denotes the kernel of

$$
A^{\bullet}(W) \rightarrow A^{\bullet}(U) .
$$

Assume that $U \hookrightarrow W$ is a Lefschetz embedding, that is, $A^{\bullet}(W) \rightarrow A^{\bullet}(U)$ is surjective. Then $A^{\bullet}(U)=A^{\bullet}(W) / J_{U / W}$. Define a Chern polynomial $P_{U / W}(t)$ to be a polynomial

$$
P_{U / W}(t)=t^{d}+a_{1} t^{d-1}+\cdots+a_{d-1} t+a_{d} \in A^{\bullet} W[t],
$$

where $d$ is the codimension of $U$ in $W$ and $a_{i} \in A^{i}(W)$ is a class whose restriction in $A^{i} U$ is the Chern class $c_{i}\left(N_{U / W}\right)$, where $N_{U / W}$ is the normal bundle of $U$ in $W$. 
In addition, it is required that $a_{d}=[U]$ be the class of $U$, which is a class restricting to the top Chern class $c_{d}\left(N_{U / W}\right)$.

Lemma $4.1\left([4)\right.$. Let $\left\{U_{i}\right\}$ be disjoint smooth closed subvarieties of a smooth variety $W$. Assume that all inclusions $U_{i} \hookrightarrow W$ are Lefschetz embeddings. Then the Chow ring $A^{\bullet}\left(\mathrm{Bl}_{\left\{U_{i}\right\}} W\right)$ is isomorphic to

$$
A^{\bullet}(W)\left[T_{1}, \ldots, T_{m}\right] / I
$$

where $T_{i}$ corresponds to the exceptional divisor $\widetilde{U_{i}}$ for $U_{i}$ and $I$ is the ideal generated by

(1) $T_{i} \cdot T_{j}$ for $i \neq j$;

(2) $P_{U_{i} / W}\left(-T_{i}\right)$ for all $i$;

(3) $J_{U_{i} / W} \cdot T_{i}$ for all $i$.

Proof. When $m=1$, this is Theorem 1, Appendix of [4]. Assume the statement is true for $m=r$. Consider the blowup

$$
\mathrm{Bl}_{\left\{U_{i}: 1 \leq i \leq r+1\right\}} W \rightarrow \mathrm{Bl}_{\left\{U_{i}: 1 \leq i \leq r\right\}} W
$$

along the proper transform $U_{r+1}^{r}$ of $U_{r+1}$. Observe that

$$
P_{U_{r+1}^{r} / \mathrm{Bl}_{\left\{U_{i}: 1 \leq i \leq r\right\}} W}=P_{U_{r+1} / W}
$$

and

$$
J_{U_{r+1}^{r} / \mathrm{Bl}_{\left\{U_{i}: 1 \leq i \leq r\right\}} W}=\left(J_{U_{r+1} / W}, U_{1}^{r+1}, \ldots, U_{r}^{r+1}\right)
$$

where $U_{1}^{r+1}, \ldots, U_{r}^{r+1}$ are the exceptional divisors of

$$
\mathrm{Bl}_{\left\{U_{i}: 1 \leq i \leq r+1\right\}} W \rightarrow W
$$

corresponding to $U_{1}, \ldots, U_{r}$. Hence the case for $r+1$ follows from the inductive assumption and Theorem 1 (i.e., $m=1$ ), Appendix of [4].

We identify $A^{\bullet}(W)$ as a subring of $A^{\bullet}(\mathrm{Bl} W)$ by means of the injection $p^{*}$ : $A^{\bullet}(W) \rightarrow A^{\bullet}(\mathrm{Bl} W)$ where $p$ is the projection $\mathrm{Bl} W \rightarrow W$.

Lemma 4.2 ([1]). Assume that $U$ and $V$ are smooth closed subvarieties of $W$ and meet cleanly in a smooth closed subvariety $Z$.

(1) $P_{\mathrm{Bl}_{Z} U / \mathrm{Bl}_{V} W}(t)=P_{U / W}(t)$;

(2) $P_{\mathrm{Bl}_{Z} U / \mathrm{Bl}_{Z} W}(t)=P_{U / W}(t-\widetilde{Z})$ where $\widetilde{Z}$ is the exceptional divisor in $\mathrm{Bl}_{Z} W$.

Proof. This is basically Lemma 6.2 of [1] except that $U$ and $V$ meet cleanly instead of transversally.

(1) follows since $N_{\mathrm{Bl}_{Z} U / \mathrm{Bl}_{V} W}$ is the pull-back of $N_{U / W}$.

(2) follows since $N_{\mathrm{Bl}_{Z} U / \mathrm{Bl}_{V} W}=\left.p^{*}\left(N_{U / W}\right) \otimes \mathcal{O}(-\widetilde{Z})\right|_{\mathrm{Bl}_{Z} U}$ and the verification used in [1], where $p$ is the restriction to $\mathrm{Bl}_{Z} U$ of the map $\mathrm{Bl}_{Z} W \rightarrow W$.

Lemma 4.3 ([1]). Assume that $U$ and $V$ are smooth closed subvarieties of $W$ and meet cleanly in a smooth closed subvariety $Z$. Assume also that $Z \hookrightarrow U, V \hookrightarrow W$ are all Lefschetz embeddings. Then all the relevant inclusions below are Lefschetz embeddings, and

(1) $J_{\mathrm{Bl}_{Z} U / \mathrm{Bl}_{V} W}=J_{U / W}$ if $Z \neq \emptyset$;

(2) $J_{\mathrm{Bl}_{Z} U / \mathrm{Bl}_{V} W}=\left(J_{U / W}, \tilde{V}\right)$ if $Z=\emptyset$, where $\tilde{V}$ is the exceptional divisor in $\mathrm{Bl}_{V} W$ 
(3) $J_{\mathrm{Bl}_{Z} U / \mathrm{Bl}_{Z} W}=\left(J_{U / W},\left[\mathrm{Bl}_{Z} V\right]\right)$ if $Z \neq \emptyset$. Note that $\mathrm{Bl}_{Z} V$ is the proper transform of $V$.

Proof. (1) and (2) together are Lemma 6.4 of [1].

(3). By Lemma 6.5 of [1], $J_{\mathrm{Bl}_{Z} U / \mathrm{Bl}_{Z} W}=\left(J_{U / W}, P_{V / W}(-\widetilde{Z})\right)$. By Lemma 4.2 (2), $P_{V / W}(-\widetilde{Z})=P_{\mathrm{Bl}_{Z} V / \mathrm{Bl}_{Z} W}(0)=\left[\mathrm{Bl}_{Z} V\right]$.

Proof of Theorem 1.8. First, we fix some notation. Let $\mathcal{D}_{=r}:=\left\{D_{r, 1}, \ldots, D_{r, l_{r}}\right\}$ be the subset of rank $r$ elements of the arrangement $\mathcal{D}$.

We now prove the corresponding statement for $\mathrm{Bl}_{\mathcal{D}_{\leq r}} X$ by using induction on $r$.

When $r=0$, this follows directly from Lemma 4.1.

Assume that the statement is true for $\mathrm{Bl}_{\mathcal{D}_{\leq r}} X$; i.e. the Chow ring $A^{\bullet}\left(\mathrm{Bl}_{\mathcal{D}_{\leq r}} X\right)$ is isomorphic to the polynomial ring

$$
A^{\bullet}(X)\left[T_{1, l_{1}}, \ldots, T_{r, l_{r}}\right] / I_{r} .
$$

Here $T_{m, j}$ corresponds to the exceptional divisor $E_{m, j}^{m+1}$ (see Theorem 1.7](1) for the description of $E_{m, j}^{m+1}$ ) for $D_{m, j} \in \mathcal{D}_{\leq r}$ and $I_{r}$ is the ideal generated by

(1) $T_{m, i} \cdot T_{n, j}$ for incomparable $D_{m, i}$ and $D_{n, j}$ where $m, n \leq r$;

(2) $P_{D_{m, i} / X}\left(-\sum_{D_{n, j} \leq D_{m, i}} T_{n, j}\right)$ for all $(m, i)$ where $m \leq r$;

(3) $J_{D_{m, i} / X} \cdot T_{m, i}$ for all $i$ where $m \leq r$.

(Each variable $T_{i}$ geometrically corresponds to the cycle of the explicit exceptional divisor $E_{i}^{r+1}$ in the blowup stage $\mathrm{Bl}_{\mathcal{D}_{\leq r}} X \rightarrow X$ where $r=\operatorname{rank}\left(D_{i}\right)$. This has to be kept in mind in the course of the rest of the proof.)

Consider now the blowup $\mathrm{Bl}_{\mathcal{D}_{\leq 0}^{r+1}}\left(\mathrm{Bl}_{\mathcal{D}_{\leq r}} X\right) \rightarrow \mathrm{Bl}_{\mathcal{D}_{\leq r}} X$. By Lemma 4.1 $A \bullet\left(\mathrm{Bl}_{\mathcal{D}_{\leq r+1}} X\right)$ is isomorphic to the polynomial ring

$$
A^{\bullet}\left(\mathrm{Bl}_{\mathcal{D}_{\leq r}} X\right)\left[T_{r+1,1}, \ldots, T_{r+1, l_{r+1}}\right] / I_{r+1}^{\prime}
$$

where $T_{r+1, i}$ corresponds to the exceptional divisor $E_{r+1, i}^{r+2}$ for $D_{r+1, i}$ of rank $r+1$ and $I_{r+1}^{\prime}$ is generated by

(1) $T_{r+1, i} \cdot T_{r+1, j}$ for $i \neq j$;

(2) $P_{D_{r+1, i}^{r+1} / \mathrm{Bl}_{\mathcal{D}_{\leq r}} X}\left(-T_{r+1, i}\right)$ for all $i$;

(3) $J_{D_{r+1, i}^{r+1} / \mathrm{Bl}_{\mathcal{D}_{\leq r}} X} \cdot T_{r+1, i}$ for all $i$.

For relation (2), we have

$$
P_{D_{r+1, i}^{r+1} / \mathrm{Bl}_{\mathcal{D}_{\leq r} X}}\left(-T_{r+1, i}\right)=P_{D_{r+1, i} / X}\left(-\sum_{D_{m, j} \leq D_{r+1, i}} T_{m, j}\right) .
$$

This is because by using Lemma 4.2 (1) and (2) repeatedly

$$
\begin{aligned}
& P_{D_{r+1, i}^{r+1} / \mathrm{Bl}_{\mathcal{D}_{\leq r}} X}\left(-T_{r+1, i}\right) \\
& =P_{D_{r+1, i}^{r} / \mathrm{Bl}_{\mathcal{D}_{\leq r-1}} X}\left(-T_{r+1, i}-\sum_{D_{r, j}<D_{r+1, i}} T_{r, j}\right) \\
& =\ldots=P_{D_{r+1, i} / X}\left(-\sum_{D_{m, j} \leq D_{r+1, i}} T_{m, j}\right) .
\end{aligned}
$$

For (3), we have by Lemma 4.3 (2) and (3) that $J_{D_{r+1, i}^{r+1} / \mathrm{Bl}_{\mathcal{D}_{\leq r} X}}$ is generated by

$$
J_{D_{r+1, i}^{r} / \mathrm{Bl}_{\mathcal{D}_{\leq r-1}}} X, T_{r, j}, D_{r+1, l}^{r+1}
$$


where $D_{r, j}$ and $D_{r+1, i}$ are incomparable and $D_{r+1, i} \cap D_{r+1, l}=D_{r, h}$ for some $h$. Here $T_{r, j}$ comes from Lemma 4.3 (2), while the proper transform $D_{r+1, l}^{r+1}$ is due to Lemma 4.3 (3). But by the projection formula, the relation $D_{r+1, l}^{r+1} \cdot T_{r+1, i}$ follows from relation (1) $T_{r+1, l} \cdot T_{r+1, i}$ and is thus redundant. Hence, $I_{r+1}^{\prime}$ is generated by

(1) $T_{r+1, i} \cdot T_{m, j}$ for incomparable $D_{r+1, i}$ and $D_{m, j}, r \leq m \leq r+1$;

(2) $P_{D_{r+1, i} / X}\left(-\sum_{D_{m, j} \leq D_{r+1, i}} T_{m, j}\right)$ for all $i$;

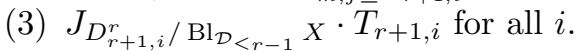

The same argument as above used again shows that $J_{D_{r+1, i}^{r}} / \mathrm{Bl}_{\mathcal{D}_{\leq r-1} X}$ is generated by

$$
J_{D_{r+1, i}^{r-1} / \mathrm{Bl}_{\mathcal{D}_{\leq r-2}}}, T_{r-1, j}, D_{r, l}^{r}
$$

where $D_{r-1, j}$ and $D_{r+1, i}$ are incomparable and $D_{r+1, i} \cap D_{r, l}=D_{r-1, h}$ for some $h$. Note that $D_{r+1, i}$ and $D_{r, l}$ are necessarily incomparable. Again, the relation $D_{r, l}^{r} \cdot T_{r+1, i}$ follows from $T_{r, l} \cdot T_{r+1, i}$ by the projection formula and is therefore redundant. This reduces the relations above to

(1) $T_{r+1, i} \cdot T_{m, j}$ for incomparable $D_{r+1, i}$ and $D_{m, j}, r-1 \leq m \leq r+1$;

(2) $P_{D_{r+1, i} / X}\left(-\sum_{D_{m, j}<D_{r+1, i}} T_{m, j}\right)$ for all $i$;

(3) $J_{D_{r+1, i}^{r-1} / \mathrm{Bl}_{\mathcal{D}_{\leq r-2}} X} \cdot \bar{T}_{r+1, i}$ for all $i$.

By repeating this procedure, we will eventually achieve that $I_{r+1}^{\prime}$ is generated by

(1) $T_{r+1, i} \cdot T_{m, j}$ for incomparable $D_{r+1, i}$ and $D_{m, j}$ where $m \leq r+1$;

(2) $P_{D_{r+1, i} / X}\left(-\sum_{D_{m, j} \leq D_{r+1, i}} T_{m, j}\right)$ for all $i$;

(3) $J_{D_{r+1, i} / X} \cdot T_{r+1, i}$ for all $i$.

Combining this with the inductive assumption on the case for $r$, the case for $r+1$ follows.

The statement of the theorem is the case when $r=\operatorname{rank}$ of $\mathcal{D}$.

The same results, with essentially the same proof, hold for $H^{*}\left(\mathrm{Bl}_{\mathcal{D}} X\right)$.

As pointed out in [1], once an open variety $X^{0}$ is campactified by adding normal crossing divisors, there is a standard way to construct a differential graded algebra $\left(\mathcal{A}^{\bullet}, \mathrm{d}\right)$. A general theorem of Morgan asserts that $\left(\mathcal{A}^{\bullet}, \mathrm{d}\right)$ is a model for the space $X^{0}$. Thus $\left(\mathcal{A}^{\bullet}, \mathrm{d}\right)$ determines the rational homotopy type of $X^{0}$. In particular, $H^{*}\left(\mathcal{A}^{\bullet}\right)=H^{*}\left(X^{0}\right)$. This provides a practical approach to compute, for example, the cohomology of the complements of arrangements in $\mathbb{C}^{n}$ or $\mathbb{P}^{n}$.

\section{Hodge Polynomial of $\mathrm{Bl}_{\mathcal{D}} X$}

For any quasiprojective variety $V$ there is a (virtual Hodge) polynomial $\mathbf{e}(V)$ in two variables $u$ and $v$ which is uniquely determined by the following properties:

(1) If $V$ is smooth and projective, then $\mathbf{e}(V)=\sum h^{p, q}(-u)^{p}(-u)^{q}$.

(2) If $U$ is a closed subvariety of $V$, then $\mathbf{e}(V)=\mathbf{e}(V \backslash U)+\mathbf{e}(U)$.

(3) If $V \rightarrow B$ is a Zariski locally trivial bundle with fiber $F$, then $\mathbf{e}(V)=$ $\mathbf{e}(B) \mathbf{e}(F)$.

The virtual Poincaré polynomial $\mathbf{P}(V)$ is defined similarly.

Proof of Theorem [1.9. We use induction on the number of elements in the arrangement of the subvarieties.

When $|\mathcal{D}|=1$, it is standard. Assume that the formula is true when the number of elements in the arrangement of the subvariety is less than $|\mathcal{D}|$. 
From the blowup

$$
\mathrm{Bl}_{\mathcal{D}} X=\mathrm{Bl}_{\mathcal{D}_{\leq 0}^{k}}\left(\mathrm{Bl}_{\mathcal{D}_{\leq k-1}} X\right) \rightarrow \mathrm{Bl}_{\mathcal{D}_{\leq k-1}} X
$$

and the descriptions of the proper transforms of $D_{i}$ (i.e., Theorem 1.4), we have

$$
\mathbf{e}\left(\mathrm{Bl}_{\mathcal{D}} X\right)=\mathbf{e}\left(\mathrm{Bl}_{\mathcal{D}_{\leq k-1}} X\right)+\sum_{\operatorname{rank}\left(D_{i}\right)=k} \mathbf{e}\left(\mathrm{Bl}_{D_{<D_{i}}} D_{i}\right) \frac{(u v)^{\operatorname{dim} X-\operatorname{dim} D_{i}}-u v}{u v-1} .
$$

By the inductive assumption,

$$
\mathbf{e}\left(\mathrm{Bl}_{\mathcal{D}_{\leq k-1}} X\right)=\mathbf{e}(X)+\sum_{\substack{D_{i_{1}}<\ldots<D_{i_{r+1}} \\ D_{i_{r+1}}:=X}} \mathbf{e}\left(D_{i_{1}}\right) \prod_{j=1}^{r} \frac{(u v)^{\operatorname{dim} D_{i_{j+1}}-\operatorname{dim} D_{i_{j}}-u v}}{u v-1}
$$

where each $D_{i_{j}}$ is of rank $\leq k-1$, and

$$
\mathbf{e}\left(\mathrm{Bl}_{D_{<D_{i}}} D_{i}\right)=\mathbf{e}\left(D_{i}\right)+\sum_{\substack{D_{i_{1}}<\ldots<D_{i_{r}} \\ D_{i_{r}}:=D_{i}}} \mathbf{e}\left(D_{i_{1}}\right) \prod_{j=1}^{r-1} \frac{(u v)^{\operatorname{dim} D_{i_{j+1}}-\operatorname{dim} D_{i_{j}}-u v}}{u v-1} .
$$

The formula in Theorem 1.9 then follows from a direct computation from here. Note that the convention $D_{i_{r+1}}:=X$ in the index of summation is a manipulation to make the formula uniform and concise.

When $u v$ is substituted by $t^{2}$, the essentially same proof yields the formula for Poincaré polynomials.

Proofs of Corollaries 1.10 1.13. Corollary 1.10 is immediate from Theorem 1.9.

Corollary 1.11 follows from Theorem 1.9 but needs a little manipulation of indexes to absorb the extra term $\mathbf{e}\left(\mathbb{P}^{n}\right)$ into the summation as indexed by " $\emptyset<X$ ". The index " $\emptyset<H_{i_{1}}$ " is used the same way for the factor $\mathbf{e}\left(H_{i_{1}}\right)$.

Corollary 1.12 follows directly from 1.11. The convention $\left|S_{i_{r+1}}\right|:=n+1$ in the index is for a unified look of the factors in the product. (Note that a recursive formula for the Betti numbers of $\bar{M}_{0, n}$ was calculated by Keel via a different sequence of blowups [4].)

Corollary 1.13 is a special case of Theorem 1.9. A similar manipulation of indexes as for Corollary 1.11 is used.

\section{SPACE OF MAPS $\mathbb{P}^{1} \rightarrow \mathbb{P}^{n}$}

From the introduction, $N_{d}\left(\mathbb{P}^{n}\right)$ is the space of equivalence classes of $(n+1)$ tuples $\left(f_{0}, \ldots, f_{n}\right) \neq 0$ where the $f_{i}$ are homogeneous polynomials of degree $d$ in two variables, and

$$
\left(f_{0}, \ldots, f_{n}\right) \sim\left(f_{0}^{\prime}, \ldots, f_{n}^{\prime}\right)
$$

if $\left(f_{0}^{\prime}, \ldots, f_{n}^{\prime}\right)=c\left(f_{0}, \ldots, f_{n}\right)$ for some constant $c \neq 0$.

Let $\mathbf{1}_{d}$ be the top partition $1+1+\ldots+1$ of $d$ and $\mathbf{0}_{d}$ the bottom (non)partition $d$ of $d$. If $\tau$ and $\tau^{\prime}$ are partitions of $d$, let $\tau \vee \tau^{\prime}$ be the least partition that both $\tau$ and $\tau^{\prime}$ proceed. $\tau\left(\mathbf{1}_{r}\right)$ denotes the partition $\tau+\mathbf{1}_{r}$ of $d+r . \rho(\tau)$ denotes the number of integers in the partition $\tau$.

Let $W^{(d)}$ be the $d$-th symmetric product of a variety $W$. Then we should have

(1) $N_{d_{0}, \tau} \cap N_{d_{0}, \tau^{\prime}}=N_{d_{0}, \tau \vee \tau^{\prime}}$;

(2) $N_{d_{0}, \tau} \cap N_{d_{0}^{\prime}, \tau^{\prime}}=N_{d_{0}^{\prime}, \tau\left(\mathbf{1}_{d_{0}-d_{0}^{\prime}}\right) \vee \tau^{\prime}}$ if $d_{0}>d_{0}^{\prime}$. 
(1) can be reduced to the configuration of unordered $\left(d-d_{0}\right)$ points in $\mathbb{P}^{1}$. There, the result is clear.

(2) follows from the observation that by factoring $d_{0}-d_{0}^{\prime}$ linear factors from degree $d_{0}$ polynomials we obtain

$$
N_{d_{0}, \tau} \cap N_{d_{0}^{\prime}, \tau^{\prime}}=N_{d_{0}^{\prime}, \tau\left(\mathbf{1}_{d_{0}-d_{0}^{\prime}}\right)} \cap N_{d_{0}^{\prime}, \tau^{\prime}}
$$

Hence it is reduced to $(2)$.

As a poset, one can check the following partial relation: $N_{d_{0}, \tau}>N_{d_{0}^{\prime}, \tau^{\prime}}$ if one of the following holds:

(1) $d_{0}=d_{0}^{\prime}, \tau>\tau^{\prime}$

(2) $d_{0}>d_{0}^{\prime}, \tau\left(\mathbf{1}_{d_{0}-d_{0}^{\prime}}\right) \geq \tau^{\prime}$.

Unfortunately, it can be checked that $N_{d_{0}, \tau}$ may in general have singularities along lower strata.

The poset $\mathcal{N}$ has the smallest element $N_{0, \mathbf{0}_{d}}$. This is a smooth subvariety in $N_{d}\left(\mathbb{P}^{n}\right)$. The strata of rank 2 have singularities along this subvariety, in general. However, it is possible that after blowing up $N_{0, \mathbf{0}_{d}}$, the singularities of the strata of rank 2 get resolved and their proper transforms become separated so that the blowups along these proper transforms can be carried out. Of course, the proper transforms of the strata on level 3 may still have singularities along the proper transforms of the strata of level 2; the hope is that after blowing up the proper transform of the strata of level 2, they too get resolved and become separated so that the process can be carried on.

This requires an intensive analysis of singularities of the strata and the effects of blowups on them. But the problem seems very interesting and of independent value, and calls for immediate investigation.

If the above turns out to be true, then one can still compute the Hodge numbers.

For a partition $\tau$, let $\rho(\tau)$ be the number of integers in the partition. Then the same method applied earlier would give that the polynomials $\mathbf{e}\left(\mathrm{Bl}_{\mathcal{N}} N_{d}\left(\mathbb{P}^{n}\right)\right)$ and $\mathbf{P}\left(\mathrm{Bl}_{\mathcal{N}} N_{d}\left(\mathbb{P}^{n}\right)\right)$ are given respectively by

$$
\begin{aligned}
& \sum_{\left(d_{i_{0}}, \tau_{i_{0}}\right),\left(d_{i_{0}}^{\prime}, \tau_{i_{0}}^{\prime}\right)<\left(d_{i_{1}}, \tau_{i_{1}}\right) \ldots<\left(d_{i_{r+1}}, \tau_{i_{r=1}}\right)} \prod_{j=0}^{r} \frac{(u v)^{(n+1)\left(d_{i_{j+1}}-d_{i_{j}}\right)+\rho\left(\tau_{i_{j+1}}\right)-\rho\left(\tau_{i_{j}}\right)}-u v}{u v-1}, \\
& \begin{array}{c}
d_{i_{0}}:=-1, \tau_{i_{0}}:=\tau_{i_{1}} \\
d_{i_{0}}^{\prime}:=d_{i_{1}}, \tau_{i_{0}}^{\prime}:=\emptyset
\end{array} \\
& d_{i_{r+1}}:=d, \tau_{i_{r+1}}:=\emptyset
\end{aligned}
$$

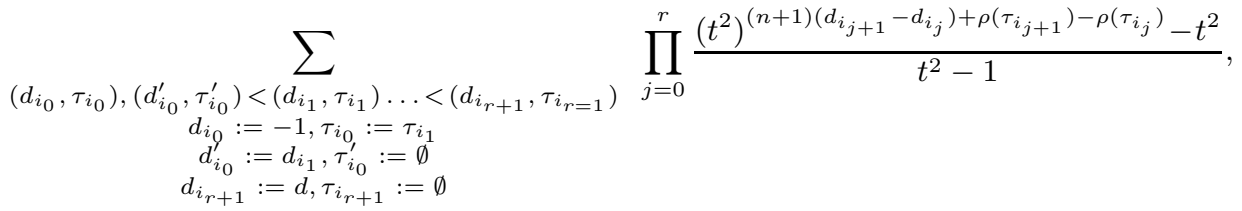

where $\rho(\emptyset):=0$.

If $X$ is embedded in $\mathbb{P}^{n}$ such that the closure $N_{d}(X)$ in $N_{d}\left(\mathbb{P}^{n}\right)$ of the space $N_{d}^{0}(X)$ of holomorphic maps of degree $d$ from $\mathbb{P}^{1}$ to $X$ is an orbifold and meets nicely with the subvarieties $N_{d_{0}, \tau}$ of the above arrangement $\mathcal{N}$, then the blowup $\mathrm{Bl}_{\mathcal{N}} N_{d}\left(\mathbb{P}^{n}\right)$ might induce a blowup of $N_{d}(X)$ and a nice projective compactification $\mathrm{Bl}_{\mathcal{N}(X)} N_{d}(X)$ of $N_{d}^{0}(X)$ by adding normal crossing divisors, where $\mathcal{N}(X)$ is the arrangement of the subvarieties that are intersections of $N_{d}(X)$ and $N_{d_{0}, \tau}$. We wonder to what extent this is the case for homogeneous spaces or more generally convex varieties. 
Replacing the two variables $\left(w_{0}, w_{1}\right)$ by multiple variables, a formal extension of the results to maps $\mathbb{P}^{m} \rightarrow \mathbb{P}^{n}$ may be possible.

\section{Partial desingularization of Git quotients}

In this section, the base field is assumed to be of characteristic 0 .

Let a reductive algebraic group $G$ act algebraically on a smooth projective variety $X$. Let $L$ be a linearized ample line bundle over $X$ and let $X^{s}=X^{s}(L)\left(X^{s s}=\right.$ $\left.X^{s s}(L)\right)$ be the open subset of (semi)stable points in $X . X^{s s} \backslash X^{s}$ may not be empty.

By replacing $L$ by a large tensor power, we may assume that $L$ is very ample and hence induces an equivariant embedding $X \hookrightarrow \mathbb{P}^{n}$.

Let $\Re$ be the set of conjugacy classes of all connected reductive subgroups of $G$ and let $R$ be a representative of an arbitrary class in $\Re$. Define

$$
Z_{R}^{s s}=\left\{\left[x_{0}, \ldots, x_{n}\right] \in X \mid\left(x_{0}, \ldots, x_{n}\right) \text { is fixed by } R\right\} \cap X^{s s} .
$$

Then $G Z_{R}^{s s}$ is a closed smooth subvariety in $X^{s s}$ by 5.10 and 5.11 of [5]. Different $Z_{R}^{s s}$, as connected components of reductive subgroups, meet cleanly. So do the corresponding $G Z_{R}^{s s}$ by 5.10 of [5].

Lemma 7.1. Let $\mathcal{R}$ be the set $\left\{G Z_{R}^{s s}: R \in \Re\right\}$. Then $\mathcal{R}$ is an arrangement of smooth subvarieties of $X^{s s}$.

Note that the above statement is void when $X^{s s}=X^{s}$.

Lemma 7.2. Let $W$ be a smooth algebraic variety acted on by a reductive algebraic group $G$. Let $W^{0}$ be a G-invariant open subset such that $W \backslash W^{0}=\bigcup_{i} D_{i}$ is a union of smooth $G$-invariant divisors $D_{i}$ with normal crossings. Assume that $L$ is a linearization of the $G$-action such that $W^{s s}=W^{s}$. Then $W^{s} / G \backslash\left(W^{s} \cap W^{0}\right) / G=$ $\bigcup_{i}\left(W^{s} \cap D_{i}\right) / G$ is a union of normal crossing divisors with at worst finite quotient singularities 3 (Note that some $\left(W^{s} \cap D_{i}\right) / G$ may be empty.)

Proof. It suffices to check that $\left\{\left(W^{s} \cap D_{i}\right) / G\right\}_{i}$ meet transversally. This is a local question.

Given a point $x \in W^{s}$, by Luna's étale slice theorem, there is a $G$-invariant open neighborhood $W_{x}$ of the point $x$ in $W^{s}$ and a smooth $G_{x}$-invariant subvariety $S_{x}$ in $W_{x}$ containing $x$ such that $W_{x}=G \cdot S_{x}$ and the natural map

$$
G \times{ }_{G_{x}} S_{x} \rightarrow G \cdot S_{x}=W_{x}
$$

is étale. Here $G_{x}$ is the finite isotropy subgroup of $G$ at $x$. This induces étale maps

$$
G \times_{G_{x}}\left(S_{x} \cap D_{i}\right) \rightarrow G \cdot\left(S_{x} \cap D_{i}\right)=\left(G \cdot S_{x}\right) \cap D_{i}=W_{x} \cap D_{i}
$$

where $S_{x} \cap D_{i}$ is either empty or a divisor in $S_{x}$. Now, since the $\left\{D_{i}\right\}$ meet transversally in $W_{x}$, we have that their corresponding $\left\{D_{i} \cap S_{x}\right\}$ meet transversally in $S_{x}$. Hence the quotients $\left\{\left(S_{x} \cap D_{i}\right) / G_{x}\right\}$ by the finite group $G_{x}$, as divisors with at worst finite quotient singularities, meet transversally by definition (see footnote 3). Finally, we can use the natural identification $\left(W_{x} \cap D_{i}\right) / G \cong\left(S_{x} \cap D_{i}\right) / G_{x}$ (local analytically) to conclude the proof.

\footnotetext{
${ }^{3}$ Such divisors are said to meet transversally if up to a finite etalé covering they meet transversally in the usual sense.
} 
Let $\mathcal{D}$ be any arrangement of $G$-invariant subvarieties in $X^{s s}$ such that $\mathcal{D}$ contains $\mathcal{R}$ as a subarrangement. Let $E$ be the exceptional divisors of $p: \mathrm{Bl}_{\mathcal{D}} X^{s s} \rightarrow X^{s s}$. $M_{d}=p^{*}\left(L^{\otimes d}\right) \otimes \mathcal{O}(-E)$ admits a linearization and $\left(\mathrm{Bl}_{\mathcal{D}} X^{s s}\right)^{s s}\left(M_{d}\right)$ is independent for sufficiently large $d$. In the following, the stability and quotient are taken with respect to the linearization $M_{d}$ for a fixed sufficiently large $d$. By Lemma 6.1 of [ [5], $\left(\mathrm{Bl}_{\mathcal{R}} X^{s s}\right)^{s s}=\left(\mathrm{Bl}_{\mathcal{R}} X^{s s}\right)^{s}$. Then by relative GIT (e.g., Theorems 3.11 and 4.4 of [2]), $\left(\mathrm{Bl}_{\mathcal{D}} X^{s s}\right)^{s s}=\left(\mathrm{Bl}_{\mathcal{D}} X^{s s}\right)^{s}$.

Corollary 7.3. The variety $\mathrm{Bl}_{\mathcal{D}} X^{\text {ss }}$ has a geometric quotient such that the following diagram is commutative:

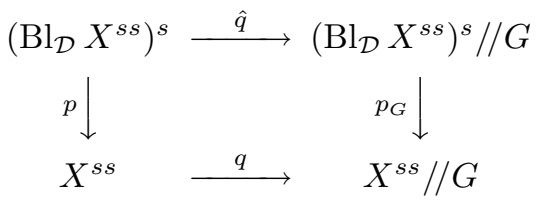

and the complement of $\left(\hat{q} \circ p^{-1}\left(X^{s} \cap X^{0}\right)\right) / / G \cong\left(X^{s} \cap X^{0}\right) / / G$ in $\left(\mathrm{Bl}_{\mathcal{D}} X^{s s}\right)^{s} / / G$ is a union of normal crossing divisors with at worst finite quotient singularities, where $X^{0}=X^{s s} \backslash \bigcup \mathcal{D}$. Moreover,

$$
p_{G}:\left(\mathrm{Bl}_{\mathcal{D}} X^{s s}\right)^{s} / / G \rightarrow X^{s s} / / G
$$

is a blowup along the induced arrangement of the images of the subvarieties in $\mathcal{D}$.

Proof. This follows from the combination of Theorem 1.1 and Proposition 6.9 of [5]. One needs to observe that each stage of the blowups $p: \mathrm{Bl}_{\mathcal{D}} X^{s s} \rightarrow X^{s s}$ yields (by applying Lemma 3.11 of [5]) a corresponding stage of blowups $p_{G}$ : $\left(\mathrm{Bl}_{\mathcal{D}} X^{s s}\right)^{s} / / G \rightarrow X^{s s} / / G$ and once $D_{i}$ and $D_{j}$ get separated in a certain stage the same becomes true for their images in $X^{s s} / / G$.

Note that the blowup $p_{G}$ is not covered by Theorem 1.1 due to the presence of singularities. But the blowing up procedure and the reason that it can be carried out is essentially the same, as indicated in the above proof.

When $\mathcal{D}=\mathcal{R}$, we recover Kirwan's partial desingularization of $X^{s s} / / G([5])$.

\section{ACKNOWLEDGEMENTS}

Our paper clearly follows the ideas and methods of some earlier works, especially those of Ulyanov [7] and MacPherson and Procesi [6]. The computation of the intersection ring follows that of Fulton and MacPherson [1]. I thank them all. I am very grateful to Professor Fulton for his instructive comments and generous advice. I thank MPI in Bonn for financial support (summer 1999) while this paper was being written. I am very indebted to Professor S.-T Yau for his valuable support and Professor S. Keel for pointing out a serious mistake. The research is partially supported by NSF and NSA.

\section{ADDED IN PROOF}

Works related to the topics of this paper can also be found in:

C. De Concini and C. Procesi, Wonderful models of subspace arrangements, Selecta Math. (N.S.) 1 (1995), no. 3, 459-494.

Eduard Looijenga, Compactifications defined by arrangements I: The ball quotient case, math.AG/0106228. 


\section{REFERENCES}

1. W. Fulton and R. MacPherson, A compactification of configuration spaces, Ann. of Math. 139 (1994), 183-225. MR 95j:14002

2. Y. Hu, Relative Geometric Invariant Theory and Universal Moduli Spaces, International Journal of Mathematics Vol. 7 No. 2 (1996), 151-181. MR 98i:14016

3. $\mathrm{Y} . \mathrm{Hu}$, Moduli spaces of stable polygons and symplectic structures on $\overline{\mathcal{M}}_{0, n}$, Compositio Mathematicae 118 (1999), 159-187. MR 2000g:14018

4. S. Keel, Intersection theory of moduli spaces of stable pointed curves of genus zero, Transactions AMS. 330 (1992), 545-574. MR 92f:14003

5. F. Kirwan, Partial desingularization of quotients of nonsingular varieties and their Betti numbers, Annals of Math. 122 (1985), 41-85. MR 87a:14010

6. R. MacPherson and C. Procesi, Making conical compactifications wonderful, Selecta Math. (N.S.) 4 (1998), no. 1, 125-139. MR 2001b:32032

7. A. Ulyanov, A polydiagonal compactification of configuration spaces, J. Algebraic Geom. 11 (2002), 129-159. MR 2002j:14004

Department of Mathematics, University of Arizona, Tucson, Arizona 85721

E-mail address: yhu@math.arizona.edu 